\title{
Ship leadership, situation awareness and crew safety behaviour - Pre-registered replications in two survey datasets
}

\author{
Bjørn Sætrevik ${ }^{1,2} \&$ Sigurd W. Hystad 1,3 \\ 1: Operational Psychology Research Group, Faculty of Psychology, University of Bergen, Christies gate 12, NO-5015 Bergen. \\ 2: Email: bjorn.satrevik@,uib.no, corresponding author \\ 3: Email: sigurd.hystad@,uib.no
}

\begin{abstract}
Situation awareness is often assumed to be central for safety work. A self-reported context-general measure of situation awareness allows us to test it in large datasets and analyse its relationships with other variables relevant for safety. A previous study (Sætrevik \& Hystad, 2017, Safety Science) found that authentic leadership predicted situation awareness and self-report of committing unsafe actions. Additionally, situation awareness predicted subjective risk assessment and unsafe actions. The current study performed pre-registered replication analyzes of the associations between authentic leadership, situation awareness, unsafe actions, and risk assessment in two novel survey datasets using structural equation models. Both datasets replicated the associations between situation awareness and unsafe actions, and between situation awareness and subjective risk assessment. The dataset that measured leadership found it to be associated with both situation awareness and unsafe actions. The pre-registered structural equation models explained large amounts of the variance in situation awareness and unsafe actions, and medium to large amounts of the variance in subjective risk assessment. We also tested adjusted models that incorporated more of the measured items and improved the validity of the measures. The study also supports the claim that a crewmember's cognitive states are associated with safety outcomes, and that leadership qualities may facilitate this relationship. This pre-registered replication in two novel datasets demonstrates the reliability of the previously identified relationships.
\end{abstract}

\section{Keywords}

Authentic leadership, situation awareness, safety management, unsafe actions, offshore supply vessel, pre-registration, replication, structural equation modelling

\section{1: Introduction}

Perception and understanding of the immediate environment form the basis for making decisions about safety-critical behaviour. The leadership in an organization may have transformative effects on the followers' motivation and framework for understanding their environment. In the analysis of a survey dataset collected in 2013 (Sætrevik \& Hystad, 2017), we found that crewmembers that reported that their captain provided authentic leadership (AL) reported higher levels of situation awareness (SA). AL and SA was also associated with seeing their work as more dangerous and that they committed more unsafe actions in their daily work. In the current study, we seek to replicate the same associations in two novel similar datasets collected in 2015 and 2017.

\section{1: Situation awareness}

Situation awareness (SA) has been suggested to be a crucial sharp-end indicator for safety across a number of safety settings (Endsley, 1995b). SA is often understood as the cognitive process and the resulting cognitive states involved for an operator to engage with their environment in order to make better decisions. SA thus involves making a mental model of the safety-critical aspects of the work, which then forms the basis of assessment and decision-making. Although both the definition and theoretical framework of SA is subject to discussion and is in competition with other concepts (Dekker, Hummerdal, \& Smith, 2010; Sarter \& Woods, 1991), it remains an influential concept among researchers and practitioners. The dominant theoretical model describes three different levels of SA where level one concerns the perception of relevant features in your environment, level two concerns the understanding of the environment, and level three concerns anticipating how the environment will develop in the near future.

$\mathrm{SA}$ is traditionally measured in relation to a given taskenvironment (Patrick \& Morgan, 2010), by providing the operator with a given scenario to perform and subsequently measuring the operator's actions (process measure), the accuracy of their beliefs (objective measure) or self-reported performance (subjective measure). We have previously argued (Sætrevik, 2013) that it may also be of value to measure to which extent the operator feels that they have a sufficient overview of safety-critical aspects across different workcontexts (a context-general measure of SA). It is this measure that was used in our previous study (Sætrevik \& Hystad, 2017), and that will be replicated here.

\section{2: Predictors of situation awareness}

As $\mathrm{SA}$ is assumed to form the basis for actions, research has attempted to identify factors that may facilitate the development of accurate and suitable SA for various environments (Endsley, 2016; Jentsch, Salas, Sellin-Wolters, $\&$ Bowers, 1995). As SA is often explored in specific taskenvironments, the research tends to explore the impact of various task or environment manipulations (such as implementing a new control interface), rather than of individual, inter-individual or organizational factors. Thus aspects related to the task or work-environment, availability of necessary information, work-load, length of work shifts, time-of-day, etc. have been explored (Sandhåland, Oltedal, Hystad, \& Eid, 2015; Sneddon, Mearns, \& Flin, 2013). These findings have consequences for workplace design and organization, but may have limited transfer value across different work settings. 
From a psychology perspective, it becomes apparent that the concept of SA overlaps with cognitive constructs, such as perception, schema, mental models, and mental simulation, sense-making and decision-making. These concepts build on a generalized cognitive ability that predicts performance in a number of job contexts (Ree, Earles, \& Teachout, 1994; Schmidt \& Hunter, 2004). Based on this, one may assume that individual variation in a crewmember's ability to perceive, understand and make sense of their environments also has a predictive value for specific work situations.

The work's organization, leadership and interpersonal interaction may influence the operator's capacity to develop SA. Most safety-critical work is done in teams (CannonBowers \& Salas, 2016), and social environments provide information, emphasis and a framework for understanding risks and safe behaviour. In small teams where some of the work is done individually (such as on an offshore supply vessel), the team leader may have an important role in enabling the other team members to develop SA). There has been considerable research on the effect of leadership on safety in recent years (Clarke, 2013; O'Dea \& Flin, 2001). Safety leadership has been found to be associated with safety climate, safety participation and safety compliance. In particular, transformational leadership styles may be effective in motivating employee participation in safety.

An authentic leader is aware of his or her strengths and weaknesses, and openly shares these with others (Avolio \& Gardner, 2005; Gardner, Cogliser, Davis, \& Dickens, 2011). Further, AL is characterized by soliciting opposing viewpoints and objectively considering all relevant information before reaching a conclusion. The decisions of authentic leaders are guided by their moral standards, instead of blindly complying with outside pressures. Because authentic leaders are aware of how their own behaviour and decisions will set important standards and influence others, they are believed to act as positive role models for safety at work (Sandhåland et al., 2015). On the offshore service vessels studied here, AL may influence and motivate the crew to attend to safety in their work.

\section{3: Safety outcomes of situation awareness}

The safe operation of complex socio-technical systems, such as offshore service vessels, relies on a number of interacting factors. In comparable systems, skill-based slips or memory lapses have been associated with creating risk (Hobbs \& Williamson, 2002; Reason, 1990; Rothblum, 2000; Sheridan, 2008). Thus the accuracy of a crewmember's SA may influence whether they are aware of safety-critical information and how their actions influence safety in their daily work. This will impact both their motivation and their ability to work safely. Work in the hydrocarbon energy sector is to a large extent regulated by safety procedures and explicit expectations for safety attitudes and behaviours. As a consequence of this, one may expect the crewmembers to have a clear idea of when their work is in compliance with the safety regulations and when they are taking unwanted risks. Our previous study (Sætrevik \& Hystad, 2017) had as an outcome measure of safety the crewmembers' self-report of the extent to which they engaged in various unsafe actions at work, in the sense of "cutting corners" in their adherence to the safety procedures. Previous studies have shown that committing unsafe actions is associated with increased risk of accidents and unwanted incidents (Hobbs \& Williamson, 2002; Hofmann \& Stetzer, 1996). This is not to say that identifying "human error" or "loss of SA" is a satisfactory level of explanation for why accidents happen, as their underlying causes can be further explored and may present better targets for interventions.

The crewmembers' impression of the level of safety on board will reflect not only the risks caused by their own actions, but also the actions of their co-workers, technical conditions, and risks that are seen as random or uncontrollable. The crew's subjective assessment of the level of risk could to some extent reflect the actual safety (Flin, Mearns, Gordon, \& Fleming, 1996; Kirschenbaum, Oigenblick, \& Goldberg, 2000; Sneddon et al., 2013), although the relationship may be complex and the causal direction unclear (Rundmo, 1996). Based on this, our previous study also asked the respondents about the extent to which they perceive their work as dangerous, in terms of estimating the risk of being involved in an accident in the subsequent year. We may expect that a crewmember that perceives and understands the safety-critical information will see their workplace as safer, and that they are less at risk for an accident. While this does not directly address whether actual risk is associated with SA, previous studies in comparable settings have indicated that inaccurate mental models may precede accidents (Endsley, 1995a; Sneddon, Mearns, \& Flin, 2006).

\section{4: The analysis to be replicated}

The aim of the current study was to replicate the findings from our previous study (Sætrevik \& Hystad, 2017). That study analysed survey responses collected in 2013 from 705 crewmembers on 49 offshore supply vessels operating on the Norwegian Continental Shelf on a number of variables related to safety. A structural equations model (SEM) analysis found support for the following hypotheses: That crewmembers that reported to have more accurate SA performed fewer unsafe actions (H1), and saw risk for accidents to be lower $(\mathrm{H} 2)$, and that reporting that the captain provided more AL was associated with fewer unsafe actions (H3) and more accurate SA (H4). As risk assessment and unsafe actions were both measured by self-report at the same time and may have response overlap, we did not expect to be able to discern meaningful causal mechanisms between them in the current studies, and thus had no hypothesized relationship between them.

\section{5: Aims of the current study}

While most research build on past studies to test previously identified relationships, they often use different settings for data collection, different measures or different analytical approaches (i.e. they are conceptual replications, Yong, 2012). However, with such approaches there is no way of saying how similar the methods and results need to be in order to say that a phenomenon has been replicated, there may be disputes when conceptual replications produce opposing results, and vested authors may be tempted to claim that a result has been confirmed in the case of positive results, while negative results are discounted as being due to methodological differences. Direct replications (Simons, 2014) try to test 
previously identified relationships in a way that is as similar as possible to the previous study. This allows us to state with more confidence whether a phenomenon is reliable, to separate true phenomena from methodological or situational artefacts, and will further the scientific discourse. In order to provide transparency and to show that the various analysis choices were not informed by the data (Gelman \& Loken, 2013), or exploited the researcher's degrees of freedom (Simmons, Nelson, \& Simonsohn, 2011), studies should be pre-registered, in terms of providing a description of the planned study, hypotheses and analysis in as much detail as possible, that is time-stamped ahead of data collection or the analysis.

Our previous study (Sætrevik \& Hystad, 2017) was based on analysis of data collected in 2013. Subsequent to this, similar data collections were made for ships on hire for the same company in 2015 and 2017, allowing for full or partial replication of the analysis. A pre-registration was made on Open Science Framework (OSF; https://osf.io/bcu6f/), which described the hypotheses, data collection, analysis plan and inference criteria. At the time that the registration was made, the 2015 dataset had been collected and subjected to various descriptive analyses, but the relationships described in the registration had not been tested. The 2017 dataset was in the process of being collected at that time, but had not been compiled to a data file.

The following pre-registered hypotheses will be tested, corresponding to the hypotheses in the previous study (Sætrevik \& Hystad, 2017): SA will be negatively associated with "unsafe actions" (H1). SA will be negatively associated with "subjective risk assessment" (H2). AL will be negatively associated with "unsafe actions" (H3). AL will be positively associated with SA (H4). As AL was not measured in 2017, $\mathrm{H} 3$ and H4 will only be tested in the 2015 dataset. In addition, we will explore various adjustments to the statistical models, and measurement of the variables, some of which were anticipated in the pre-registration. As different measures were used in 2015 and 2017, and the two datasets are expected to have partially overlapping samples, the two datasets will be analysed separately. The OSF folder (https://osf.io/bcu6f/) contains the pre-registration, surveys, item text for each variable, data sets, analysis syntax and output.

\section{2: Materials and methods}

\section{1: Data collection}

The 2015 data collection was done in same way as in the tobe replicated data collection from 2013 (Sætrevik \& Hystad, 2017). A survey booklet was distributed to all employees of maritime vessels operating on the Norwegian Continental Shelf on hire for a single hydrocarbon energy company. The crewmembers returned the surveys directly to the researchers individually or through the company mail.

The surveys were distributed to 37 vessels, with 30 surveys going to each vessel. There were 444 surveys with valid responses returned from 27 of the vessels. In accordance with pre-registration, scores for items with negative phrasing were reversed, and 39 participants that had reported non-Nordic nationalities or failed to report their nationality were removed, retaining 405 participants.
In the 2013 survey the captains had evaluated the leadership style of their immediate leader in the organization, and responses from the captains were thus excluded from the analysis of the captain's leadership style. In the 2015 survey, captains were asked to assess their own leadership styles. Although it was not specified in the pre-registration how to handle this discrepancy, we decided to remove the captains from the sample, as the captains' own assessment of their leadership styles would be causally different from the crewmembers' assessment of their captain's leadership. We thus removed 34 captains from the 2015 sample, retaining 371 participants.

The 2017 data collection was done in same way as in 2013 and 2015 , but with separate survey forms for captains. There were 555 valid surveys returned from 34 vessels. In accordance with the pre-registration, we removed 38 surveys where the respondent stated a non-Nordic nationality or failed to answer the question, retaining 517 participants. To maintain similarity with the 2013 and 2015 analyses, we removed 48 surveys where the respondent stated to be the captain or failed to answer the question, retaining 469 participants.

\section{2: Measures in the 2015 dataset}

AL and SA were measured in the same way in 2015 as in 2013. AL was measured using the 16 item "Authentic leadership questionnaire" (ALQ, Walumbwa, Avolio, Gardner, Wernsing, \& Peterson, 2008), which had been translated into Norwegian by the first author. SA was measured with the 13 item "context-general situation awareness" scale (Sætrevik, 2013).

Unsafe actions were measured with the same seven items as in the 2013 dataset. The items described actions that could increase the likelihood of accidents, or actions that are in conflict with the safety procedures. In order to create parcels for the SEM analysis, a principal component factor analysis (PCA) was performed on the seven items measured in 2015, fixed to extract 3 factors using a varimax rotation. Based on the factor loadings and attempting to balance the items equally between the three factors, we set the first parcel to consist of items 120,122, and 126, the second parcel to consist of items 123 and 124, and the third parcel to consist of items 121 and 125 .

Subjective risk assessment was measured with the same seven items as in 2013, where the risks for various types of accidents are graded from 1 to 8 (going from "no risk" to "a large risk"; types of accidents extracted from Norwegian Maritime Authority, 2011). In accordance with the preregistration, an additional item (not measured in 2013) was included that rated the risk of major accidents. A PCA was performed across the eight items in the scale, with a fixed number of 3 factors to extract, using a varimax rotation. This resulted in assigning item 199, 206, and 227 to the first parcel, item 220, 234, and 246 to the second parcel, and item 213 and 241 to the third parcel.

\section{3: Measures in the 2017 dataset}

AL was not measured in 2017, and the variable was therefore removed from the statistical models of this dataset. SA was measured with the same 13 items as in 2013 and 2015. 


\section{5 data}

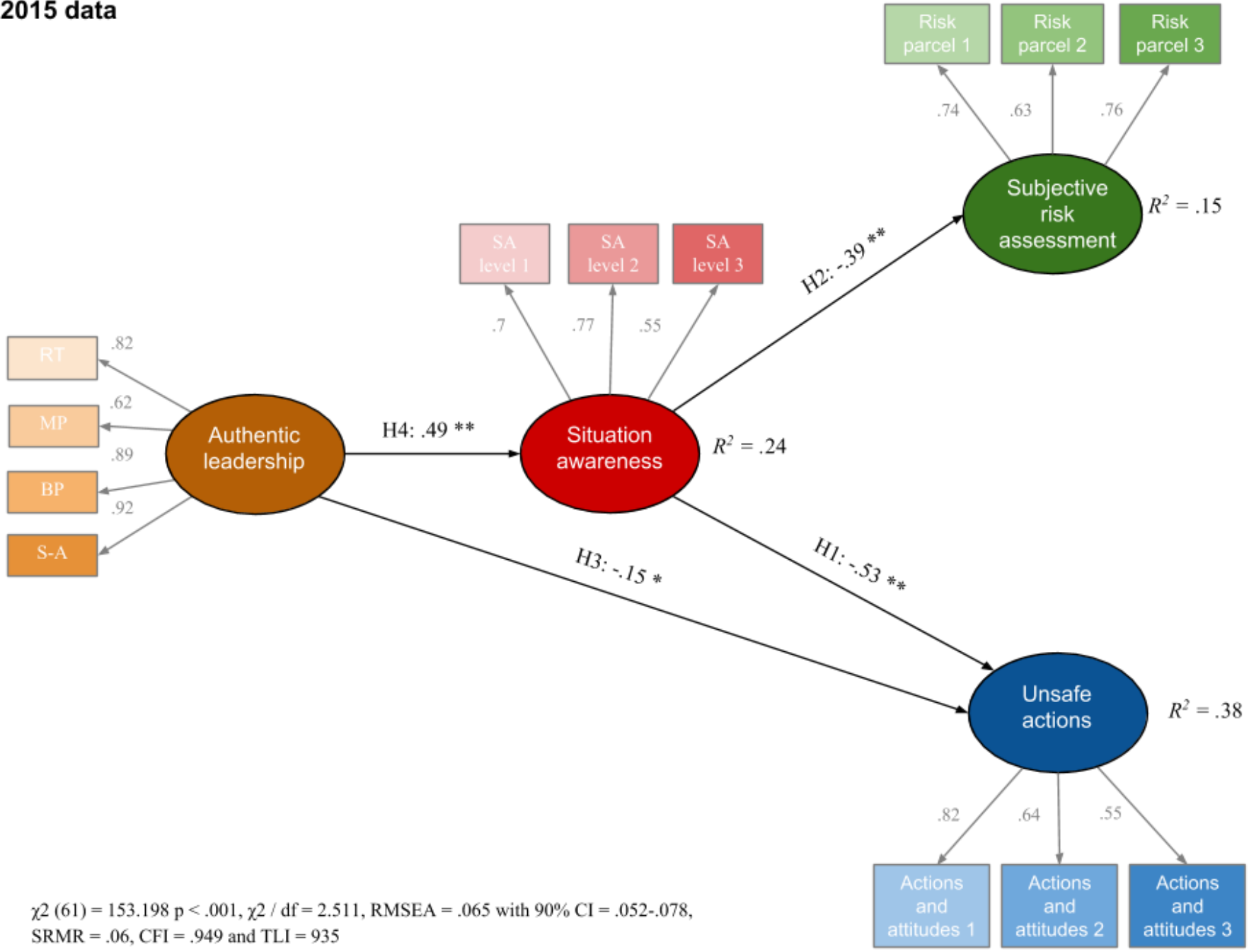

Figure 1. Standardized coefficients for the pre-registered structural model on the 2015 dataset. Factor loadings marked with ** are significant at $p<.001$, while the H3 association marked with * is significant at $p=.02$ (one-tailed).

As described in the pre-registration, "unsafe actions" was measured with three new items in addition to the seven items used in 2015. One of the new items was suggested by our industry partners (159: "I do not have enough time to use the safety tools properly"), and two items are from the Brief NORSCI scale (Nielsen, Eid, Hystad, Saetrevik, \& Saus, 2013; 116: "I urge colleagues to stop work that I believe is being carried out in a risky way" and 117: "I stop work if I think it may be dangerous for me or others to continue"). In order to create parcels for the SEM analysis, a PCA was performed across all three datasets on the 10 items, fixed to extract 3 factors, using a varimax rotation. Based on this we set the first parcel to consist of items 121,125, 126 and 159, the second parcel to consist of item 116, 123, and 124, and the third parcel to consist of item 117, 120 and 122. This also revealed that item 159 had negative loading to all factors. An evaluation of this item's face validity also indicated that it had a poor fit to the overall concept, and should be removed in the explorative analyses (see section 5.3).

Subjective risk assessment was measured with two novel items, asking about the risk for being injured in an accident (in general, rather than asking about different accident types as in 2013 and 2015), and one item asking about the risk for being involved in a major accident.
See full item text for all variables in all three datasets in the OSF folder (https://osf.io/bcu6f/).

\section{3: Results}

\section{1: Measurement model in 2015}

The structural model described AL to be associated with SA and "unsafe actions", SA to be associated with "unsafe actions" and "subjective risk". A confirmatory factor analysis (CFA) showed good fit for the model's subfactors and parcels of the latent variables, and covariates between the latent variables $\left(\chi^{2}(59)=134.003 \mathrm{p}<.001, \chi^{2} / \mathrm{df}=2.271\right.$, RMSEA $=.06$ with $90 \% \mathrm{CI}=.046-.073, \mathrm{SRMR}=.05, \mathrm{CFI}=$ .958 and TLI $=945)$.

\section{2: Pre-registered latent variable model in 2015}

The pre-registered structural model for the 2015 dataset (see Figure 1 below) tested H1, H2, H3 and H4 as similarly as possible to the test performed on the 2013 dataset (Sætrevik \& Hystad, 2017). The model showed good fit indices, and model fit, factor loadings and explained variance were similar to the 2013 data, thus indicating a direct replication of these findings. There were medium to strong associations from SA to "unsafe actions" (H1) and to "subjective risk assessment" $(\mathrm{H} 2)$, and between AL and SA (H4), while the association between AL and "unsafe actions" was weaker (H3). The 
model accounted for large amounts of the variance for all three latent variables.

\section{3: Adjusting the 2015 "unsafe actions" latent variable}

The pre-registered analysis described "unsafe actions" as consisting of seven items in order to maintain similarity to the previously published analysis (Sætrevik \& Hystad, 2017). However, the pre-registration also argued for removing three of the items (121, 123 and 125), as they could be said to describe "attitudes" rather than "actions" (for example item 121: "Safety is not the most important aspect of my work"). Adjusting the model accordingly resulted in mostly unchanged factor loadings (H1: -.51, p < .001, H2: -.39, p < .001 , H3: -.15, $\mathrm{p}=.022$, one-tailed, H4: -.49, $\mathrm{p}<.001$ ), explained variance $\left(\mathrm{SA}: \mathrm{r}^{2}=.24\right.$, "subjective risk assessment": $\mathrm{r}^{2}=.16$, "unsafe actions": $\left.\mathrm{r}^{2}=.35\right)$, and goodness of fit indices $\left(\chi^{2}(50)=147.534, \mathrm{p}<.001, \chi^{2} / \mathrm{df}=2.95\right.$, RMSEA $=.074$ with $90 \% \mathrm{CI}=.06-.088, \mathrm{SRMR}=.065, \mathrm{CFI}=.944$ and TLI = 926). Despite the limited consequences of this, we find that this adjustment of the "unsafe actions" variable to be preferable as it could be argued to increase validity.

\section{4: Adjusting "subjective risk assessment" latent variable}

The pre-registered analysis modelled "subjective risk assessment" as eight items about the perceived likelihood of getting involved in seven different types of accidents (in the same way as in 2013) and in major accidents. However, in the 2015 dataset there were also additional items that asked the participant to assess the risk of the same seven types of accidents befalling someone else on the crew. As it could be argued that these items are also relevant for the theoretical construct of risk assessment, a revised explorative model used the sum of the eight items from the pre-registered analysis as a first observed indicator for "subjective risk assessment", while the sum of the seven additional items were added as a second observed indicator. The model implementing this adjustment as well as the adjustment of the "unsafe actions" variable described above (section 3.3) can be seen in Figure 2. Compared to the pre-registered model in Figure 1, this model produced comparable factor loadings and explained variance for latent variables, higher loadings for the observed indicators for "subjective risk assessment", and comparable goodness of fit indices. Including the non-Nordic respondents or the captains in the sample led to small changes in model fit, association strength, and explained variance. The data, syntax and results for these analyses are available in the project folder (https://osf.io/bcu6f/).

\section{5 data, modified model}

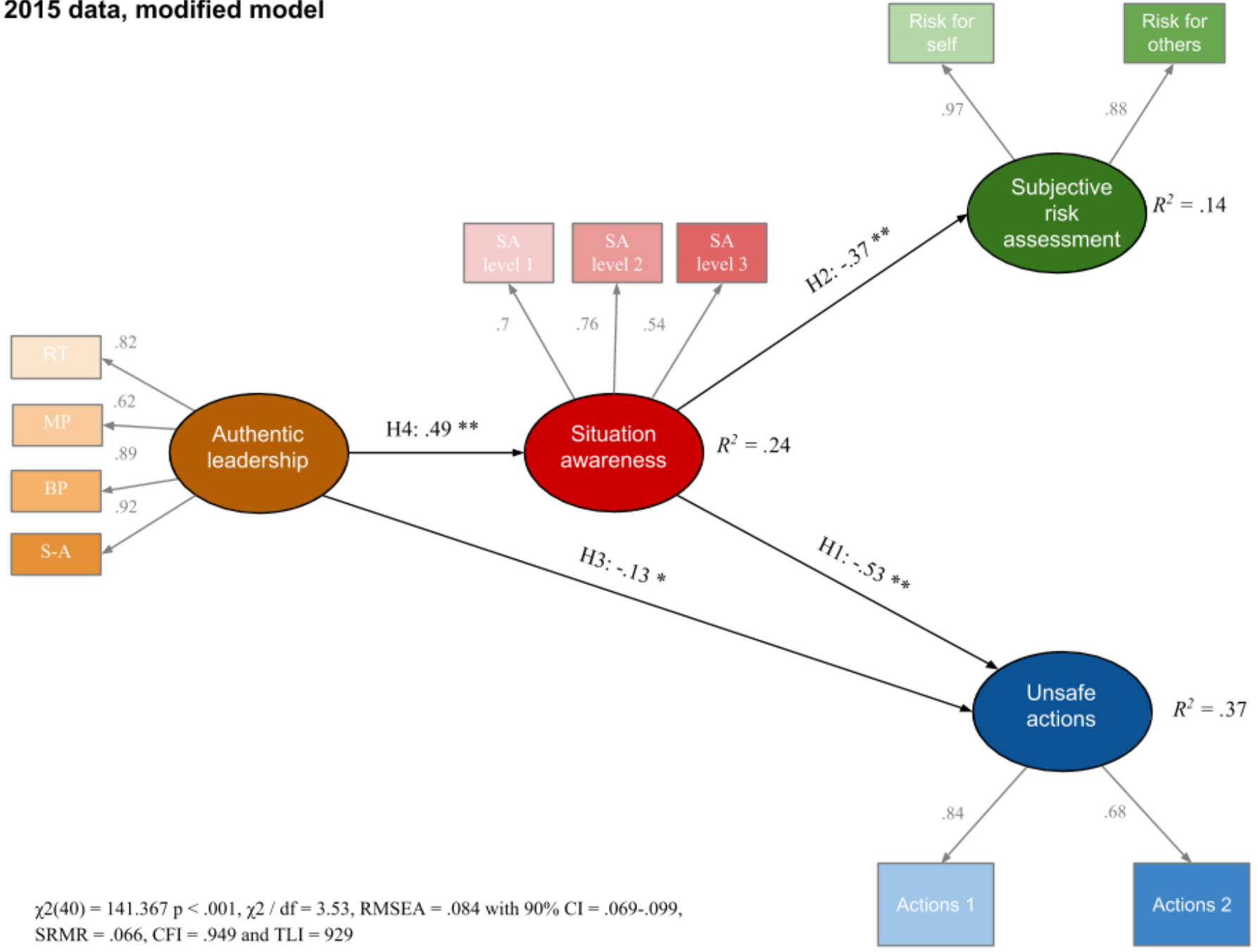

Figure 2. Standardized coefficients for an adjusted structural model applied on the 2015 dataset. Factor loadings marked with ** are significant at $p<.001$, while the H3 association marked with $*$ is significant at $p=.038$ (one-tailed). 


\section{7 data}

$\chi 2(18)=49.966 \mathrm{p}<.001, \chi 2 / \mathrm{df}=2.78, \mathrm{RMSEA}=.063$ with $90 \% \mathrm{CI}=.042-.084$,

$\mathrm{SRMR}=.058, \mathrm{CFI}=.971$ and $\mathrm{TLI}=.955$

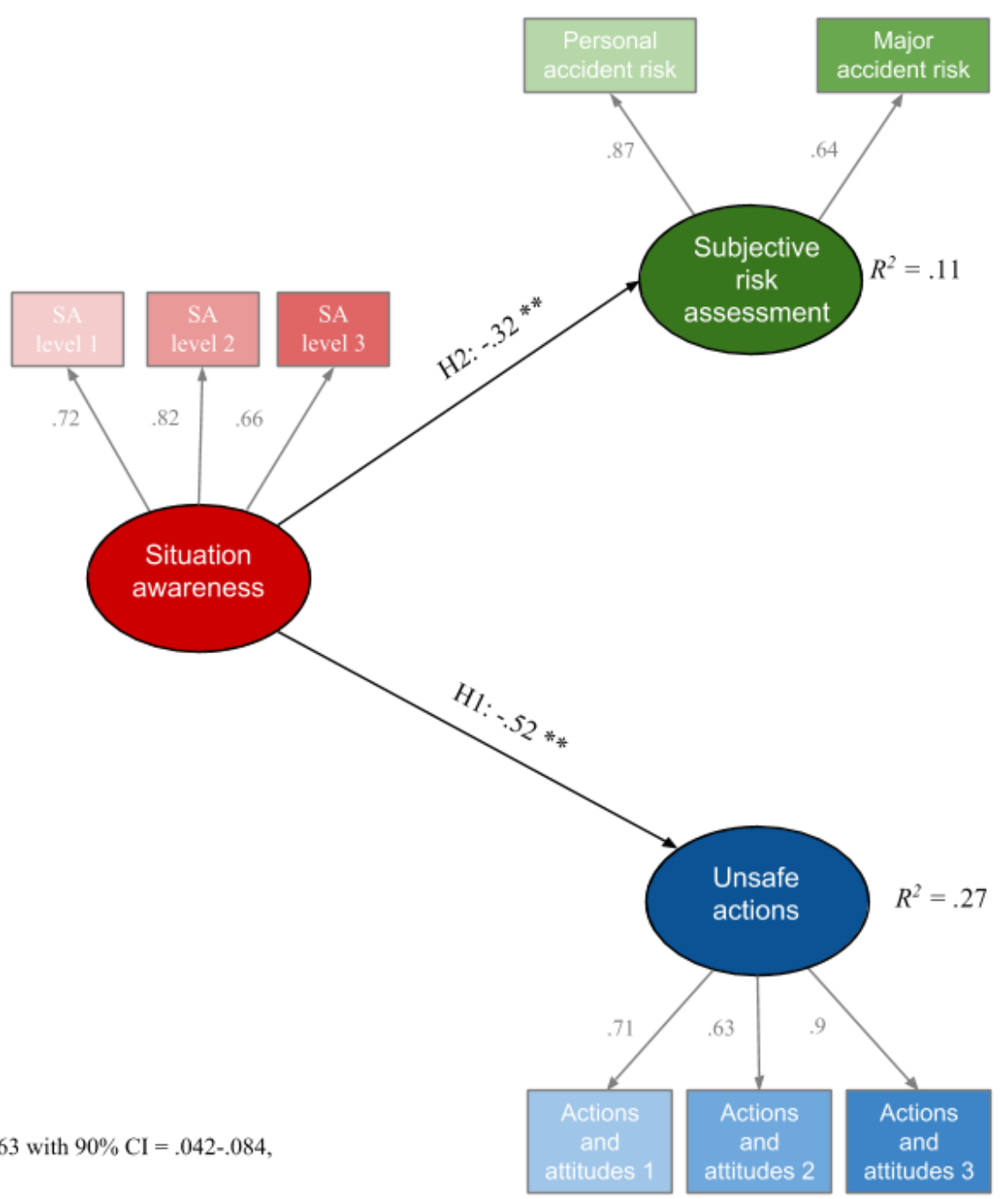

Figure 3. Standardized coefficients for the pre-registered structural model on the 2017 dataset. Both factor loadings are marked with ** to indicate significance at $p<.001$.

\section{5: Measurement model in 2017}

The structural model described SA to be associated with "unsafe actions" and "subjective risk". A CFA showed good fit for the structural model's subfactors and parcels of the latent variables, and covariates between the latent variables $\left(\chi^{2}(17)=26.12 \mathrm{p}=.015, \chi^{2} / \mathrm{df}=1.88, \mathrm{RMSEA}=.044\right.$ with $90 \% \mathrm{CI}=.019-.068, \mathrm{SRMR}=.033, \mathrm{CFI}=.986$ and TLI $=$ $.977)$.

\section{6: Pre-registered latent variable model in 2017}

The pre-registered structural model for the 2017 dataset included tests for $\mathrm{H} 1$ and $\mathrm{H} 2$, while $\mathrm{H} 3$ and $\mathrm{H} 4$ could not be tested in this dataset, as AL was not measured in 2017. (see Figure 3 below). The model found support for both the H1 and $\mathrm{H} 2$ hypotheses, which showed that SA was strongly associated with having fewer "unsafe actions" and somewhat lower with "subjective risk assessment". The model accounted for large amounts of the variation in "unsafe actions", and medium to large amount of variation in "subjective risk assessment". Although this pre-registered model shows acceptable fit indices and confirms the hypotheses being tested, we will proceed to test adjusted models for the 2017 dataset in a similar way as was been done for the 2015 dataset above. The data, syntax and results for these analyses are available in the project folder (https://osf.io/bcu6f/).
3.7: Adjusting the 2017 "unsafe actions" latent variable

Similar as for the 2015 dataset and as mentioned in the preregistration, the model was adjusted by removing three "attitude" items (121, 123 and 125) from the "unsafe actions" latent variable. In addition, as argued above we also remove item 159 due to poor coherence and validity. These adjustments resulted in increased factor loading on $\mathrm{H} 1$ (-.55, $\mathrm{p}<.001$ ), and increased explained variance for "unsafe actions" $\left(\mathrm{R}^{2}=.3\right)$. The adjusted model fit was somewhat poorer than the pre-registered model $(\chi 2(18)=68.5, \mathrm{p}<.001$, $\chi^{2} / \mathrm{df}=3.81$, RMSEA $=.079$ with $90 \%$ CI $=.06-.099$, $\mathrm{SRMR}=.056$, CFI $=.812$ and $\mathrm{TLI}=918$ ).

\section{8: Adjusting the 2017 "risk assessment" latent variable}

As was done for the 2015 dataset, an additional indicator where crewmembers assessed the risk for others on their crew to be injured was added to the "subjective risk assessment" latent variable in the 2017 analysis. As can be seen in Figure 4 , this adjustment, along with the adjustment described above (section 5.3) slightly improved factor loadings while explained variance remained unchanged. Goodness of fit was comparable to the pre-registered model. Including the nonNordic respondents or the captains in the sample led to small changes in model fit, association strength, and explained variance. 


\section{7 data, modified model}

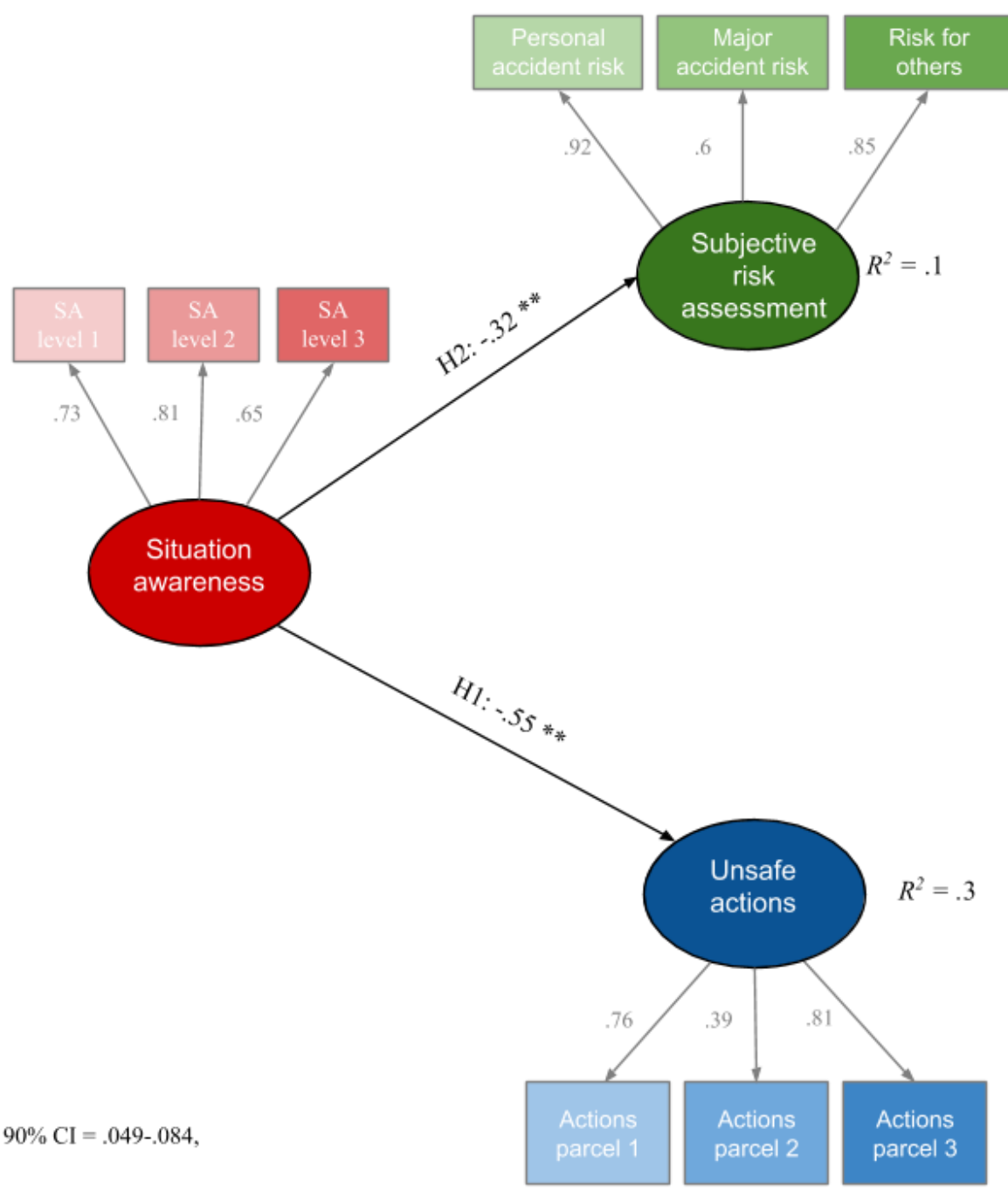

$\chi 2(25)=73.926, \chi 2 / \mathrm{df}=2.95, \mathrm{RMSEA}=.066$, with $90 \% \mathrm{CI}=.049-.084$

$\mathrm{SRMR}=.06, \mathrm{CFI}=.965$ and $\mathrm{TLI}=949$

Figure 4. Standardized coefficients for an adjusted structural equation model for the 2017 dataset. Both factor loadings are marked with ** to indicate significance at $p<.001$.

\section{4: General discussion}

\section{1: Summary of results}

The overall structural model and all the hypotheses being tested (H1, H2, H3, and H4 in 2015, H1 and H2 in 2017) were supported, and all models have acceptable model fit. There was strong support for the association of SA to "unsafe actions" and to "subjective risk assessment" (H1 and H2, both tested across both datasets), accounting for large amount of variance in "unsafe actions", and medium to large amounts of variance in "subjective risk assessment". Further, there was strong support for the association between AL and $\mathrm{SA}$, which accounted for large amount of the variance $(\mathrm{H} 4$, tested only in the 2015 dataset). There was moderate support for the association between AL and "unsafe actions" (H3, tested only in the 2015 dataset).

\section{2: Assessment of replication}

The pre-registered analyses replicated the same results as in the previously published study (Sætrevik \& Hystad, 2017) in both datasets for all four hypotheses (H3 and H4 only tested in 2015 dataset). This is despite some changes in how the two outcome variables are measured in the new datasets. This lends support to the original findings by indicating that the same relationships of similar magnitudes could be found when applying the same measurement and analyses on a similar sample two and four years later. Further, the pre- registration demonstrates that the replication is not due to ad hoc hypothesizing or researcher degrees of freedom.

While questions about "risk for other crewmembers" was not measured in 2013 and was not described in the preregistration, the model fit improved when these questions were added in both the 2015 and 2017 analyses. It is also reasonable from a theoretical evaluation that seeing the workplace as dangerous would increase both the perceived risk for oneself and the risk for others, and including these items would thus constitute a more robust measure of the concept.

As noted above, the "unsafe actions" items used in the analysis of the 2013 dataset and in the pre-registered models included items that are not strictly related to actions performed by the crewmember. Based both of face validity (as discussed in the pre-registration) and the analysis results from 2015 and 2017 indicate that these items should be removed from the construct. In addition, future measurement should use careful wording to make sure that all items are in fact associated with actions for which the crewmember is responsible, as opposed to actions that are accepted or encouraged, or actions that are performed by the crew in general, but not necessarily by the responder themselves.

Finally, while it was not specified in the pre-registration, we would recommend that when leadership is measured, the 
leaders themselves (here the captains) should be removed from the sample. This is due to the validity of the leadership measure becoming less clear if the captains are included, although follow-up analyses showed little impact of whether the captains are included or not.

\section{3: Impact of leadership and awareness on safety}

The associations that were previously identified (Sxtrevik \& Hystad, 2017) between AL, SA, "subjective risk assessment" and "unsafe actions" appear to be reliable and replicable for the current setting. The pre-registered statistical models explained large amount of the variance in the safety outcomes. This could indicate that the concepts of $\mathrm{AL}$ and SA are important for regulating risk in safety-critical organizations. This is in line with recent research indicating the primacy of mental models for maintaining safety, and how intrapersonal interaction supports the operator's work to construct accurate mental models (Hystad, Bartone, \& Eid, 2014; Nielsen, Eid, Mearns, \& Larsson, 2013).

Crew-member self-report of SA was found to be associated with and accounted for variation in risk assessment and committing unsafe actions in both the 2015 and the 2017 datasets, comparable to the effects that were seen in the 2013 dataset. High score on the study's SA measure implies that the crew experience having accurate representation of the safety critical aspects of their work on board. This would allow operators to understand which situations in the workenvironment that entail more risk and need for caution, and would facilitate decision-making in safety-critical operations. Thus SA could lead to seeing the work as more predictable and less risky, and could make it easier to work safely and comply with the safety regulations.

Crew-member's perception of the captain's AL was found to be associated with SA (only tested in the 2015 dataset), and explained large amounts of the variation in SA. AL was also associated with committing fewer unsafe actions, although this association was somewhat weaker. Again, these are the same effects as were seen in the 2013 dataset. These findings indicate that the leadership style of the immediate leader impacts the individual worker's cognitive states and actions. Although no worker wants to have an inaccurate mental model or to perform unsafe actions, in the day-to-day work these concerns are weighed against other tasks, goals, concerns, and distractions. An authentic leader may provide information and guidance, be an example, and provide motivation for the follower to pay attention and to understand the safety-critical aspects of their work, and to comply with the safety regulations. Previous research has also pointed to the role of leadership in facilitating the development of accurate mental models (see e.g. Marks, Zaccaro, \& Mathieu, 2000). In more general terms, Bass's (1985) concept of "transformational leadership" has often been associated with positive safety outcomes through aligning the followers' value systems with the safety values of the organization. This could happen through social mechanisms such as demonstrating individualized considerations, acting as a role-model, and inspiring optimism or enthusiasm, and through psychological mechanisms of identifying with the leader and work-group. Clarke (2013) argued that transformational leadership led to increased employee participation in safety, while an active transactional leadership focused on monitoring behaviour, anticipating and preventing errors from developing could increase compliance with rules and regulations.

One may note that "subjective risk assessment" was measured differently between the three datasets. Despite of this, the concept takes the same role in the structural models tested, with largely overlapping findings. This indicates a coherence between the different measures, that may reflect common underlying factors.

\section{4: Limitations and extensions}

It should be noted that the current study was performed on two independent self-report survey datasets. As both predictor and outcome variables were measured in the same way at the same time, the results may be influenced by a common method bias (Podsakoff, MacKenzie, Lee, \& Podsakoff, 2003). Thus, rather than reflecting any underlying relationship between the variables, the identified associations may be partially be due to effects such as a crewmember who had a positive and optimistic mindset on the day of answering the survey being more likely to respond that the captain is authentic, that their SA is accurate, that they see the work as safe and that they seldom violate the safety procedures. Similar criticism could be raised for much survey-based research. Nevertheless, the current findings should seek support from additional studies using other measurement approaches, such as measuring at different time points, or using multiple types of measures.

In an extension of this, it should be noted that the "unsafe actions" variable did not rely on objective measures of actions performed, but on self-report of typical behaviour patterns. This measure is thus subject to misremembering or misrepresenting the safety of one's behaviour, and to respondents comparing their behaviour to different perceived safety standards. An objective measure of behaviour in terms of process measures or observer ratings would be preferable, but is unfeasible for samples of this size.

We should note that the current studies do not use one of the traditional measure of SA. SA is often understood as the operator's perception, understanding and prediction of the available cues in a given task scenario, and is thus typically measured in relation to performing a given training exercise. Our conception of SA as a trait concept that can be measured as self-report across time and situations may thus only partially overlap with traditional uses of the term. Nevertheless, we argue elsewhere (Sætrevik, 2013) that some aspects of the concept may be trait dependent, and that the measures have a theoretical overlap. Further, the traditional measures of SA could not be included in studies like the current one.

The current study and the replicated study show correlational associations, from which we can argue there may be underlying causal mechanisms. In order to assume causality with any confidence, experiment designs should be applied. This could take the form of field-intervention studies, that examine the effects of training programs aimed at modifying the captain's leadership style, or to assist the worker's ability to understand safety aspects in their environment. 


\section{5: Conclusion}

The current study found that the previously reported associations between AL, SA, risk assessment and unsafe actions could be replicated in two additional data collections made in the same setting. Pre-registration of the analyses guarantees that researcher degrees of freedom were not exploited to replicate the results. The results indicate that the concepts of AL and SA have value in assessing safety levels on vessels and the mechanisms that may contribute to safety. They further indicate that the current measure of SA has meaningful associations with other safety variables, and can thus be of value to include in future surveys studies. Thus, we recommend that similar approaches can be used to assess safety levels and to identify features suitable for interventions to improve safety.

\section{Acknowledgements}

Data was collected in collaboration with the Logistics and Emergency preparedness department of Equinor (Development and Production Norway). Some of the survey items were developed in collaboration with subject matter experts at Equinor and at the relevant ship owner companies. The ship owner companies on hire for Equinor contributed in the data collection, and we are grateful to the vessel crews for taking time to answer the surveys.

\section{References}

Avolio, B. J., \& Gardner, W. L. (2005). Authentic leadership development: Getting to the root of positive forms of leadership. Leadership Quarterly, 16(3), 315-338. doi:10.1016/j.leaqua.2005.03.001

Cannon-Bowers, J. A., \& Salas, E. (2016). Team Performance and Training in Complex Environments. Current Directions in Psychological Science, $\quad 7(3), \quad 83-87 . \quad$ doi:10.1111/14678721.ep10773005

Clarke, S. (2013). Safety leadership: A meta-analytic review of transformational and transactional leadership styles as antecedents of safety behaviours. Journal of Occupational and Organizational Psychology, 86(1), 22-49.

Dekker, S. W. A., Hummerdal, D. H., \& Smith, K. (2010). Situation awareness: some remaining questions. Theoretical Issues in Ergonomics Science, 11(1), 131-135.

Endsley, M. R. (1995a). A taxonomy of situation awareness errors. Human factors in aviation operations, 3(2), 287-292.

Endsley, M. R. (1995b). Toward a Theory of Situation Awareness in Dynamic-Systems. Human Factors, 37(1), 32-64. doi:Doi 10.1518/001872095779049543

Endsley, M. R. (2016). Designing for situation awareness: An approach to user-centered design: CRC press.

Flin, R., Mearns, K., Gordon, R., \& Fleming, M. (1996). Risk perception by offshore workers on UK oil and gas platforms. Safety Science, 22(1-3), 131-145. doi:Doi 10.1016/0925-7535(96)00011-2

Gardner, W. L., Cogliser, C. C., Davis, K. M., \& Dickens, M. P. (2011). Authentic leadership: A review of the literature and research agenda. Leadership Quarterly, 22(6), 1120-1145. doi:10.1016/j.leaqua.2011.09.007

Gelman, A., \& Loken, E. (2013). The garden of forking paths: Why multiple comparisons can be a problem, even when there is no "fishing expedition" or "p-hacking" and the research hypothesis was posited ahead of time. Department of Statistics, Columbia University.

Hobbs, A., \& Williamson, A. (2002). Skills, rules and knowledge in aircraft maintenance: errors in context. Ergonomics, 45(4), 290-308. doi:10.1080/00140130110116100
Hofmann, D. A., \& Stetzer, A. (1996). A cross-level investigation of factors influencing unsafe behaviors and accidents. Personnel Psychology, 49(2), 307-339.

Hystad, S. W., Bartone, P. T., \& Eid, J. (2014). Positive organizational behavior and safety in the offshore oil industry: Exploring the determinants of positive safety climate. J Posit Psychol, 9(1), 42-53. doi:10.1080/17439760.2013.831467

Jentsch, F. G., Salas, E., Sellin-Wolters, S., \& Bowers, C. A. (1995). Crew coordination behaviors as predictors of problem detection and decision making times. Paper presented at the Proceedings of the Human Factors and Ergonomics Society Annual Meeting.

Kirschenbaum, A., Oigenblick, L., \& Goldberg, A. I. (2000). Well being, work environment and work accidents. Social Science \& Medicine, 50(5), 631-639. doi:Doi 10.1016/S0277-9536(99)00309-3

Marks, M. A., Zaccaro, S. J., \& Mathieu, J. E. (2000). Performance implications of leader briefings and team-interaction training for team adaptation to novel environments. $J$ Appl Psychol, 85(6), 971986.

Nielsen, M. B., Eid, J., Hystad, S. W., Saetrevik, B., \& Saus, E. R. (2013). A brief safety climate inventory for petro-maritime $\begin{array}{llll}\text { organizations. } & \text { Safety } & \text { Science, } & 58,\end{array}$ doi:10.1016/j.ssci.2013.04.002

Nielsen, M. B., Eid, J., Mearns, K., \& Larsson, G. (2013). Authentic leadership and its relationship with risk perception and safety climate. Leadership \& Organization Development Journal, 34(4), 308-325. doi:10.1108/Lodj-07-2011-0065

Norwegian Maritime Authority. (2011). Marine casualties 2000-2010. Retrieved from https://www.sdir.no/globalassets/global-2/ulykkerog-sikkerhet/ulykkesstatistikk/statistikk-ulykker/marine-casualties2000---2010.pdf

O'Dea, A., \& Flin, R. (2001). Site managers and safety leadership in the offshore oil and gas industry. Safety Science, 37(1), 39-57.

Patrick, J., \& Morgan, P. L. (2010). Approaches to understanding, analysing and developing situation awareness. Theoretical Issues in Ergonomics Science, 11(1), 41-57.

Podsakoff, P. M., MacKenzie, S. B., Lee, J. Y., \& Podsakoff, N. P. (2003). Common method biases in behavioral research: a critical review of the literature and recommended remedies. J Appl Psychol, 88(5), 879-903. doi:10.1037/0021-9010.88.5.879

Reason, J. (1990). Human error. New York: Cambridge university press.

Ree, M. J., Earles, J. A., \& Teachout, M. S. (1994). Predicting job performance: Not much more than g. Journal of Applied Psychology, 79(4), 518.

Rothblum, A. M. (2000). Human error and marine safety. Paper presented at the National Safety Council Congress and Expo, Orlando, FL.

Rundmo, T. (1996). Associations between risk perception and safety. Safety Science, 24(3), 197-209. doi:10.1016/S0925-7535(97)000386

Sandhåland, H., Oltedal, H. A., Hystad, S. W., \& Eid, J. (2015) Distributed situation awareness in complex collaborative systems: A field study of bridge operations on platform supply vessels. Journal of Occupational and Organizational Psychology, 88(2), 273-294. doi:10.1111/joop.12111

Sarter, N. B., \& Woods, D. D. (1991). Situation awareness: A critical but ill-defined phenomenon. The International Journal of Aviation Psychology, 1(1), 45-57.

Schmidt, F. L., \& Hunter, J. (2004). General mental ability in the world of work: occupational attainment and job performance. J Pers Soc Psychol, 86(1), 162-173. doi:10.1037/0022-3514.86.1.162

Sheridan, T. B. (2008). Risk, human error, and system resilience: fundamental ideas. Hum Factors, 50(3), 418-426. doi:10.1518/001872008X250773

Simmons, J. P., Nelson, L. D., \& Simonsohn, U. (2011). False-Positive Psychology: Undisclosed Flexibility in Data Collection and Analysis Allows Presenting Anything as Significant. Psychological Science, 22(11), 1359-1366. doi:10.1177/0956797611417632

Simons, D. J. (2014). The Value of Direct Replication. Perspect Psychol Sci, 9(1), 76-80. doi:10.1177/1745691613514755

Sneddon, A., Mearns, K., \& Flin, R. (2006). Safety and situation awareness: "Keeping the Bubble" in offshore drilling crews. Paper 
presented at the SPE International Health, Safety \& Environment Conference.

Sneddon, A., Mearns, K., \& Flin, R. (2013). Stress, fatigue, situation awareness and safety in offshore drilling crews. Safety Science, 56, 80-88. doi:10.1016/j.ssci.2012.05.027

Sætrevik, B. (2013). Developing a context-general self-report approach to measure three-level situation awareness. Int Marit Health, 64(2), 66-71.

Sætrevik, B., \& Hystad, S. W. (2017). Situation awareness as a determinant for unsafe actions and subjective risk assessment on offshore attendant vessels. Safety Science, 93, 214-221.

Walumbwa, F. O., Avolio, B. J., Gardner, W. L., Wernsing, T. S., \& Peterson, S. J. (2008). Authentic leadership: Development and validation of a theory-based measure. Journal of Management, 34(1), 89-126. doi:10.1177/0149206307308913

Yong, E. (2012). Replication studies: Bad copy. Nature, 485(7398), 298300. doi:10.1038/485298a 